\title{
Orthopedic Day-case Surgery in Nigeria: A Single-center Experience
}

\author{
Echezona Valentine Malizu, ${ }^{1}$ Omolade Ayoola Lasebikan, ${ }^{2}$ Njoku Isaac Omoke ${ }^{3}$ \\ ${ }^{1}$ Rehoboth Specialist Hospital, Port Harcourt, Rivers State, Nigeria \\ ${ }^{2}$ National Orthopaedic Hospital, Enugu, Enugu State \\ ${ }^{3}$ Department of Surgery, Ebonyi State University/Federal Teaching Hospital, Abakaliki, Ebonyi State, \\ Nigeria
}

Correspondence to: Dr. Njoku Omoke; email: zicopino@yahoo.com

Received: 26 August 2019; Revised: 31 March 2020; Accepted: 01 May 2020; Available online: 25 May 2020

\begin{abstract}
Background: The concept of day-case surgeries is relevant in orthopedic specialty in developing countries, where orthopedic elective procedures have relatively longer duration of surgical waiting time, mainly due to lack of inpatient bed space. We aimed to determine the scope, safety and outcome of orthopedic day-case surgeries in a Nigerian setting, and identify potential areas for intervention to improve the practice. Methods: This was a 12-month prospective study of 71 eligible, consenting and consecutive patients who presented at the National Orthopedic Hospital Enugu and were carefully selected and prepared for orthopedic day-case surgeries. Results: Within the period of study, 53 of 540 elective orthopedic procedures were carried out as daycase, giving a day-case surgery rate of $9.8 \%$. Of the patients enrolled, male to female ratio was $1.2: 1$ and age range was 8 months to 76 years. Eighteen (25.4\%) patients had their day-case procedure cancelled on the day of surgery. The commonest procedure was removal
\end{abstract}

of implant. Conversion rate was $32 \%$ mainly due to operation occurring late. Complication (mainly pain) rate was $30 \%$, and correlated with duration of procedure $(\mathrm{p}<0.006)$. The satisfaction rate among patients was $98 \%$; no re-admission or mortality was observed. Conclusion: In this study, orthopedic day-case procedures were safe, though there was low use of daycase surgery in scope, complexity and number of procedures. This and the high conversion rate observed call for a dedicated day-case unit and measures to facilitate timelines of the procedures.

Keywords: Orthopedics, Day-case surgery

Ann Afr Surg. 2021; 18(1):52-58

DOI: http://dx.doi.org/10.4314/aas.v18i1.10

Conflicts of Interest: None

Funding: None

(C) 2021 Author. This work is licensed under the Creative Commons Attribution 4.0 International License.

\section{Introduction}

Day-case surgery is an important part of elective surgery globally. It accounts for over $50 \%$ and $60 \%$ of elective surgeries in the UK and USA respectively (1). Published reports show that day-case surgery shortens hospital waiting list, facilitates efficient use of resources, and provides high-quality, safe and cost-effective surgical care in selected and well-prepared cases $(2,3,4,5)$. It is also acceptable to patients and health workers (6). The concept of day-case surgery could not be more apt in developing countries where demand for elective surgery usually outstrips inpatient facilities and long waiting list is often the norm (7). Day-case surgery is even more relevant in orthopedic specialty in developing countries. Published reports show that compared with other surgical specialties, orthopedic elective procedures have 
relatively longer duration of length of surgical waiting time mainly due to lack of inpatient bed space (8).

Safety and patient satisfaction/acceptability are two critical issues in day surgery $(6,9)$. Safety can be gauged with parameters such as the direct admission, readmission, and postoperative complication and mortality rates (9). The rates of these parameters and scope of orthopedic day-case vary from and within subregions $(2,9)$. Detailed knowledge about the scope, safety and outcome of orthopedic day-case surgery in a setting can facilitate strategies and policy response towards improving the practice. However, data is limited on orthopedic day-case surgery in developing countries; the two previous reports were retrospective studies with associated inherent limitations $(1,2)$. This underscores the importance of prospective data to evaluate orthopedic day-case surgery in our environment. Therefore, this study aimed to determine the scope, safety and outcome of orthopedic day-case surgery in a low-resource setting, and identify potential areas for intervention to improve the practice.

\section{Methods}

\section{Study setting and design}

This was a prospective descriptive study carried out among patients for orthopedic day-case surgery at the National Orthopaedic Hospital Enugu, Enugu State, Nigeria, from April 2016 to March 2017.

\section{Ethical approval}

The approval to carry out this study was obtained from the hospital's ethical committee (IRB/IIIC No. S/3131850, Protocol No. 132). A written informed consent was obtained from the patients and/or next of kin.

\section{Study population}

The study included patients of both sexes and all age groups who presented to the hospital within the stipulated study period for orthopedic day-case surgery; and satisfied the inclusion criteria.

\section{Inclusion criteria}

ASA 1 patients, ASA11 patients with controlled comorbidities, patients with hemoglobin of at least 10 $\mathrm{mg} / \mathrm{dL}$, and cases with expected duration of surgery less than 120 minutes.

\section{Exclusion criteria}

Patient living more than $30 \mathrm{~km}$ or 1-hour drive from the hospital and without relatives in town to stay with / nearby health care facility, ASA III patients, patient without responsible escort, poor domestic circumstances inappropriate for postoperative care, failure to meet inclusion criteria, patient not willing to be part of the study, and patients with uncontrolled comorbidities.

\section{Sample size}

A pilot survey of the hospital operation record book showed that in 2012 and 2013, an average of 647 elective orthopedic procedures were done (population size) and $15 \%$ of these procedures were day cases. Based on the population size and average percentage of daycase surgery in the pilot survey, a sample size of 68 was calculated from the formula: Sample size $=n /[1+\mathrm{n} /$ population $]$ where $n=[\mathrm{Z} 2 \times \mathrm{P} \times(1-\mathrm{P}) / \mathrm{D} 2](10)$.

\section{Procedure}

Patients underwent clinical assessment and laboratory investigations, hemoglobin, urinalysis and others if needed, then selected for day-case surgery. A proforma was opened for all eligible patients. Data entered included: age, sex, highest educational level attained by patient/caregiver, domicile address, estimated distance from hospital, mobile phone number, ASA grade, mode of anesthesia, procedure performed, status of surgeon, status of anesthetist, duration of surgery, time of commencement and time of end of surgery, tourniquet time (if used), access to hospital and family doctor, type of discharge analgesics, complication(s) and its duration, satisfaction of day-case surgery among patient/guardian along with reasons for satisfaction or dissatisfaction. Additional data were entered into the proforma for those that were converted (direct admission) and reasons for conversion, cases that were re-admitted and reasons for readmission, and for cases cancelled along with the reasons for cancellation. 
Conversion (direct admission) rate, otherwise called unplanned overnight admission, refers to that proportion of patients initially planned for day-case procedures who were subsequently admitted immediately after operation for any reason $(6,9)$. Re-admission rate refers to that proportion of day-cases that were operated and discharged home as planned but were admitted back within 30 days for complication developed back at home $(6,9)$.

The patients were given a detailed explanation of the objective of the study, and consent was obtained from each patient. Further explanation that patient should present in the morning of surgery with a responsible adult and would go back home after surgery was given. Patients were instructed to start fasting at midnight the night preceding surgery, using the preoperative guideline of 2 hours for clear fluid, 4 hours for breast milk and 6 hours for formula milk and solids. Patients were asked to report at theatre on the morning of surgery. At arrival in the morning and after fulfilling all administrative procedures, they were prepared for surgery by the nurse at the ward. Prophylactic antibiotic (Ceftriaxone $1 \mathrm{gm}$ and metronidazole $500 \mathrm{mg}$ ) was administered intravenously to all patients at induction of anesthesia. Patient was observed in the theatre recovery room after surgery and then moved to the ward. Patient was discharged home accompanied, after being assessed by the first author in conjunction with a senior member of the operating unit, along with satisfying the following discharge criteria: alert and oriented in time and place, stable vital signs, pain controlled by oral analgesic, nausea or emesis controlled, able to walk without dizziness, regional anesthesia appropriately resolved, prescription given, patient accepts readiness for discharge, and a responsible adult present to accompany patient home. Postoperative pain as a complication was measured at the point of discharge from hospital using numeric rating scale (NRS) $0-10$, where 0 represented no pain and 10 the severest pain intensity.

Patients were followed up through phone calls at least once a day after discharge till next clinic appointment. The patient/caregiver was also given the contact number of the first author. Patients were followed up for at least four weeks and at each clinic visit they were clinically assessed by one of the authors together with the managing unit.

\section{Data analysis}

Data were analyzed using SPSS version 20 (SPSS Chicago IL, USA) for graphs, bar charts, pie charts and frequency tables, and for cross tabulation. Continuous and categorical variables were summarized using mean, frequency, standard deviation and percentages. Mean comparison of continuous variables was done using Student's $t$ test while associations between categorical variables were done using chi square; a p-value $<0.05$ was considered significant.

\section{Results}

This study enrolled 71 consecutive eligible consenting patients for orthopedic day-case surgery. The male to female ratio was $1.2: 1$ and the age range 8 months to 76 years. The estimated distance from the patient's home to the hospital ranged from 5 to $30 \mathrm{~km}$, with a mean of 16 $\mathrm{km} \pm 7.536$.

Of the 71 patients, 18 (25.4\%) had their day-case procedure cancelled on the day of surgery, and 53 patients underwent surgery as planned. In the study period, there were 540 elective procedures and 53 of these were done as day-case, giving a day-case surgery rate of $9.8 \%$.

The three top procedures performed as day-case were removal of implants (plates and screws), biopsy, and manipulation under anesthesia (Table 1). Most (84.6\%) of the day-case surgeries were therapeutic procedures, 5 (9.6\%) were diagnostic and $3(5.8 \%)$ therapeutic/ diagnostics. All patients in this study were ASA grade 1 status. Spinal, general and local anesthesia was the mode of anesthesia given to 24 (45\%), $22(42 \%)$ and $7(13 \%)$ of patients respectively. Most $47(89 \%)$ patients were anaesthetized by nurse anesthetists; $6(11 \%)$ patients by a consultant anesthetist. The senior registrar performed $42(79 \%)$ procedures whereas a consultant orthopedic surgeon performed the remaining $11(21 \%)$.

Twelve $(22.6 \%)$ patients had access to a family doctor to care for them at home, the rest did not have family doctors. Sixteen $(30.2 \%)$ patients had private cars to access the hospital in an emergency, the rest of the 
MALIZU ET AL.

patients $(69.8 \%)$ depended on public transport to access the hospital.

Seventeen patients were admitted as in-patients after surgery, giving a conversion rate of $32 \%$. Social reasons and operation occurring too late (operation after normal working hours of $4 \mathrm{pm}$ when priority and attention of theatre workforce is focused on emergency cases) was the reason in $16(94.1 \%)$ patients that were converted to inpatient hospital admission; extensive surgical procedure was the reason in $1(5.9 \%)$ of the converted cases.

Sixteen (30\%) patients who had day-case procedure had complication. These complications were surgical related: pain and hematoma. Of these 16 patients, 15 had pain and 1 had hematoma. Hematoma was observed in a patient following excision biopsy of a popliteal mass without a wound drain. None of the patients had wound infection. There was no association between the complication and the rank of anesthetist (Table 2). The rate of complication was higher in procedures done by senior registrar than those done by the consultants but this difference was not statistically significant, $p=0.330$ (Table 2). The complication rate correlated $(\mathrm{p}<0.006)$ with mean duration of surgery (Table 3 ). There was no case of re-admission into the ward for complications developed back home, and no mortality among patients. Patients were given different types of postop analgesics: 20 were given non-steroidal anti-inflammatory drugs (NSAID), 1 had opioids, 8 NSAID+opioids, 6 NSAID+paracetamol, and 18 opioids+paracetamol. There was no significant association $(p=0.272)$ between the incidence of postoperative pain and the type of discharge analgesics. There was immediate postoperative pain in $93.8 \%$ of patients the first day postoperative; the incidence of pain reduced to $31.3 \%$ and afterward complete resolution of pain in all the patients.

The three top reasons for the cancellation of day-case procedure during the period of this study were: failure to arrive to hospital, time constraint on the part of surgeon, and lack of theatre space (Table 4). Day-case surgery was highly recommended by 52 patients, giving a satisfaction rate of $98 \%$.
Table 1: Specific procedures performed

\begin{tabular}{lc}
\hline Procedure performed & Frequency $n=53(\%)$ \\
\hline Removal of implant & $32(60.4)$ \\
Biopsy & $7(13.2)$ \\
Manipulation under anesthesia & $5(9.4)$ \\
Epidural steroid injection & $4(7.5)$ \\
Elongation of Achilles tendons & $2(3.8)$ \\
Arthrotomy & $1(1.9)$ \\
Dynamization of intramedullary nail & $1(1.9)$ \\
\hline
\end{tabular}

Table 2: Complications by rank of anesthetist and surgeon, and educational level of patient/caregiver

\begin{tabular}{lccc}
\hline & $\begin{array}{l}\text { No. with } \\
\text { complications } \\
(\%)\end{array}$ & $x^{2}$ & p-value \\
\hline Rank of anesthetist & $15(31.9)$ & 0.301 & 0.583 \\
Nurse & $1(20)$ & & \\
Consultant & & & \\
Rank of surgeon & $14(33.3)$ & 0.950 & 0.330 \\
$\quad \begin{array}{l}\text { Senior registrar } \\
\text { Consultant }\end{array}$ & $2(18.2)$ & & \\
Educational level of patient/care giver & & \\
Primary & $3(100)$ & 1.064 & 0.435 \\
Secondary & $11(73.3)$ & & \\
Tertiary & $23(65.7)$ & & \\
\hline Chi & &
\end{tabular}

$\bar{X}=$ Chi statistic

Table 3: Complication by the mean duration of surgery and tourniquet time

\begin{tabular}{lccc}
\hline & \multicolumn{2}{c}{ Complication } & p value \\
\cline { 2 - 4 } & $\begin{array}{c}\text { Yes } \\
\text { Mean } \pm \text { SD }\end{array}$ & $\begin{array}{c}\text { No } \\
\text { Mean } \pm \text { SD }\end{array}$ & \\
& $51.25 \pm 16.74$ & $5.58 \pm 19.69$ & 0.006 \\
$\begin{array}{l}\text { Duration of } \\
\text { surgery(min) } \\
\begin{array}{l}\text { Tourniquet time } \\
(\text { min })\end{array}\end{array}$ & $64.40 \pm 15.51$ & $58.50 \pm 20.82$ & 0.482 \\
\hline
\end{tabular}


Table 4: Reasons for cancellation

\begin{tabular}{lc}
\hline Reasons & Frequency (\%) \\
\hline Hospital-related & $2(11.1)$ \\
$\quad$ Lack of theatre space & $1(5.6)$ \\
Power failure & $1(5.6)$ \\
$\quad$ Faulty anesthetic machine & \\
Patient-related & $8(44.4)$ \\
$\quad$ Failure to arrive & \\
Surgeon-related & $4(22.2)$ \\
$\quad$ Time constraint & $2(11.1)$ \\
$\quad$ Unavailability of lab result & $18(100)$ \\
Total &
\end{tabular}

\section{Discussion}

The age distribution of eligible patients enrolled in this was similar to the finding reported by Ajibade et al. in another orthopedic hospital setting in northern Nigeria (2). The wide age range in this and previous studies shows availability of standard anesthesia facilities with qualified and experienced anesthetists to handle different age categories for day-case procedures in a low-resource setting such as ours $(2,11)$.

Most procedures in this study were carried out under spinal anesthesia (45\%), this was quite different from general and local anesthesia for most of the procedures in the series reported by Ajibade et al. and Adewole et al. respectively $(2,12)$. In this study, spinal anesthesia was mostly used because implant removals, the commonest procedure observed, were carried out mostly on the lower limb. Spinal anesthesia is a preferred option because the residual analgesia from block also reduces postoperative pain (4).

Therapeutic procedures constituted $84.6 \%$ of all procedures; with removal of implant (59.6\%) the commonest specific procedure performed in this study is at variance with biopsy as the commonest procedure reported by Ajibade et al. (2). The reason for this variation is not evident.

The scope of procedures in this study was similar to those reported by similar studies in other Nigerian hospitals in Kano and Lagos, though additionally Kirschner wire fixation and open reduction and internal fixation of forearm fractures with plate and screws were done as day-case procedures in Kano and Lagos respectively $(2,12)$. In Kenya, Mulimba et al. reported a wider scope and complexity of orthopedic day-case procedures; this included open reduction and internal fixation of fractures, sequestrectomies, amputations, arthroscopies of the knee, tendon and nerve repairs (12). In the United Kingdom, complex surgeries like anterior cruciate ligament reconstruction were reported by Older et al., and arthroscopic meniscectomy by Khan et al. were done as day-case $(13,14)$. Procedures such as subacromial decompression with tendon transfer and tarsal coalition excision have been reported as day-case procedures $(2,15)$. The limited development of minimally invasive orthopedic surgery, unavailability of dedicated day-case units and lack of provision of community services by community physicians and nurses in Nigeria perhaps explains the wide gap in scope of procedures in this setting and in other countries $(2,16)$.

The period of this study coincided with the peak of economic recession in Nigeria and is a plausible explanation for the rate of day-case surgery that is below the average rate observed from the pilot survey. However, the day-case surgery rate in this study was higher than the $3.48 \%$ reported by Ajibade et al. (2). This level of utilization falls short of the reported rates in other surgery specialties, such as urology (61.6\%) and plastic surgery $(37.2 \%)$, reported in Nigeria, and the reason is not evident $(14,17)$.

The conversion rate in this study was higher than $3.3 \%$, $1.07 \%, 1.4 \%$ and $0.01 \%$ reported by Ajibade et al Cardosa et al., Margorsky et al., and Mulimba et al., respectively $(2,18,19,11)$. The very high conversion rate in this study was due mainly to social reason of operation occurring too late, whereas in these previous reports, conversions were due to surgical and anesthetic reasons $(18,19,11)$. In this study, only one patient had surgical reason, extensive operation, for conversion. If late operations were eliminated, then a conversion rate of (1/53) $1.9 \%$ (resulting from extensive surgical procedure) is within the range of $2-3 \%$ recommended by the Royal College of Surgeons (19). This implies the safety of the practice in our setting and calls for measures to reduce the incidence of late operations among patients for day-case procedures. 
In this study, the mean estimated distance was $16 \mathrm{~km}$ from the hospital and only $30 \%$ of patients had private car access to the hospital, bearing in mind the relative lack of good and efficient public transport system in our setting. This is an important factor for high conversion rate: most patients cannot get back home when operations are done late, considering the security situation in our setting and therefore were admitted overnight. It is therefore important that patients are operated on at the beginning of the morning list and every effort made to ensure that day-cases are dealt with before mid-day to ensure early, safe discharge. This also calls for a dedicated day-case unit so that day-case patients will not compete with inpatients for theatre space.

The complication rate in this study was higher than $2.7 \%$ by Ajibade et al. (2) and $2.37 \%$ by Cardosa et al. in similar studies (18). Pain as the commonest complication was also similar to the findings reported by Cardosa et al. and Mulimba et al. but at variance with wound infection as commonest complication reported by Ajibade et al. $(18,11,2)$. There was no significant correlation between complication rates with mode of anesthesia, rank of surgeon, rank of anesthetist, duration of tourniquet, education level of patient/caregiver, and type of discharge analgesics, and this is similar to the findings reported by Cardosa et al. (18). There was significant correlation between duration of surgery and complication rate in this study but the reason is not evident. The resolution of pain in all patients after day one postoperation shows adequacy of our discharge analgesics and patient compliance with their intake, and ability of patient/caregiver to comply with instructions for preparation for surgery and postoperative care, as previously reported by Abdurrahman (21). That none of the patients had postoperative wound infection also shows adequacy of prophylactic antibiotics and patient/caregiver compliance with the instructions.

In this study, there was no readmission and mortality; a similar finding was reported by Adewole et al. (12). This shows proper patient selection and the safety of orthopedic day-case practice in our setting. The cancellation rate in this study was higher than $15.6 \%$ and $11.06 \%$ reported by Ramyil et al. and Kolawole et al. respectively $(5,22)$. In this study, the main reason for cancellation-failure to arrive to the hospital due to financial difficulty — was similar to the findings by Ramyil et al. but at variance with surgical-related factor of time constraint reported by Kolawole et al. $(5,22)$. An organized health insurance scheme with wide coverage may help cover the financial bills of patients. The surgeon-related factor for cancellation could be mitigated by making a realistic theatre list. A dedicated day-case unit, to eliminate day-case and inpatient competition for theatre space and surgeons' time could have prevented more than a third of the cancellations (Table 5). In this study, that over $90 \%$ of patients will recommend day-case surgery to other people is an indication of its acceptability in our environment.

\section{Conclusion}

In this study, orthopedic day-case procedures were safe, though there was low utilization of day-case surgery in scope, complexity and number of procedures. This and the high cancellation and conversion rates observed call for provision of a dedicated day-case unit and measures to facilitate timeliness of the procedures.

\section{References}

1. Abdulkareem HI. Day-case in Nigeria. Niger J Clin Pract. 2011; 14(4):383-389.

2. Ajibade J, Lawson BL, Ayeni FB. Day-case surgeryExperience from a Nigerian Orthopaedic Hospital. Ambul Surg. 2011; 17:31-35.

3. Henderson J, Goldacre MJ, Griffith M, et al. Day-case surgery: Geographical trends and readmission rate. J Epidemiol Comm Health 1989; 43:301-305.

4. Adejuyibe O, Abubakar AM, Sowande OA, et al. Day-case surgery in children in Ile-Ife, Nigeria - an audit. Niger J Surg. 1998; 5(2):60-63.

5. Ramyil VM, Dakum NK, Kidmas AT et al. Reason for day-case surgery cancellation in Jos. Niger J Surg. 2004; 10(1):17-19.

6. Baskerville PA, Jarrett PEM. Day-case inguinal hernia repair under local Anaesthetic. Ann R Coll Surg Engl. 1983; 65:224225.

7. Ifesanya AO, Ogundele O J, Ifesanya JU. Orthopaedic surgical treatment delays at a tertiary hospital in Sub-Saharan Africa: communication gaps and implications for clinical outcome. Niger Med J. 2013; 54(6):420-425.

8. Abdulkareem IH. The surgical waiting time initiative: a review of the Nigerian situation. Niger Med J.2014; 55:443-51.

9. Ojo EO, Ihezue $\mathrm{CH}$, Sule AZ, et al. The safety of day-case surgery in a developing country. J One-Day Surg. 2008; 18(1):13-18.

10. Creative Research System. Sample size formula https://www.surveysystem.com/sample-size-formula.htm Accessed 7 January 2016. 
11. Mulimba JAO, Gakuu LN, Odhiambo MA. Orthopaedics in day surgery. East Afr Orthop J 2009; 3:19-22.

12. Adewole OA, Giwa SO, Kayode MO, Shiga MO. Day-case orthopaedic surgery in a West African teaching hospital. Niger J Orthop Trauma 2008; 7(2):57-59.

13. Older J. the first four years' experience of day-case orthopaedic surgery in a district general hospital. Ann R Coll Surg Engl. 1988; 70:21-23.

14. Khan T, Jackson WF, Beard DJ, et al. The use of standard operating procedures in day-case anterior cruciate ligament reconstruction. Knee 2012; 19(4):464-468.

15. Jagodzinsk NA, Gul R, Khanum S, et al. Day surgery for major paediatric foot and ankle procedure. J Orthoped Surg (Hong Kong) 2011; 19(1):69-71.

16. Adoga AS, Onakoya PA, Mgbor NC, et al. Day-case adenotonsillectory: experience of two private clinics in Nigeria. Niger J Med. 2008; 17:296-305.
17. Ikuerowo SO, Bioku MY, Onisanyo OA, et al. Urologic daycase surgery: a five-year experience. Niger J Clin Pract. 2013; 16(1):28-30.

18. Cardosa M, Rudkin GE, Osborne GA. Outcome from day-case knee arthroscopy in a major teaching hospital. Arthroscopy. 1994; 10(6):624-633.

19. Margovsky A. Unplanned admission in day-case surgery as a clinical indicator for quality assurance. Aust NZ J Surg 2000; 70 (3):216-236.

20. Royal College of Surgeons England. Commission on the Provision of Surgical Services: Guidelines for Day-case Surgery Revised ed. Roy Coll Surg Engl. London, 1992.

21. Abdur-Rahman LO, Kolawole IK, Adeniran JO, et al. Paediatric day-case surgery: Experience from a tertiary health institution in Nigeria. Ann Afr Med. 2009; 8(3):163-167.

22. Kolawole IK, Bolaji BO. Reasons for cancellation of elective surgery in Ilorin. Niger J Surg Res. 2002; 4:28-33. 